\title{
НАУКА И ОБРАЗОВАНИЕ
}

Эл № ФС77 - 48211. Государственная регистрация №0421200025. ISSN 1994-0408

\section{Анализ области эффективного применения закиси азота} в качестве компонента топлива для двигательных установок малых космических аппаратов \# 09, сентябрь 2012

DOI: 10.7463/0912.0450400

Воронецкий А. В., Арефьев К. Ю.

УДК 621.453/.457

Россия, МГТУ им. Н.Э. Баумана voron@mx.bmstu.ru arefyev@rambler.ru

Современный уровень развития космической техники накладывает серьёзные ограничения на массогабаритные, динамические и удельные характеристики двигательных установок (ДУ) малых космических аппаратов (МКА) [1]. Некоторые из предъявляемых требований приведены в таблице 1 (данные 2006 г.).

Таблица 1. Общие требования к МКА

\begin{tabular}{|c|c|c|c|}
\hline Класс МКА & "Микро" & "Нано" & "Пико" \\
\hline Масса МКА, кг & $10-100$ & $1-10$ & $<1$ \\
\hline Оценочная стоимость, не более, млн руб. & 300 & 50 & 20 \\
\hline Энергообеспечение ДУ, Вт & $15-160$ & $6-15$ & $2-6$ \\
\hline Объем ДУ, не более, л & 60 & 4 & 0,5 \\
\hline Требуемый уровень тяги ДУ, Н & $1-500$ & $0,1-20$ & $0,01-1$ \\
\hline Полный импульс ДУ, Н*с & $100-5000$ & $10-700$ & $3-10$ \\
\hline
\end{tabular}

На современном этапе развития, наиболее востребованными являются МКА класса "Микро". С целью повышения массовой эффективности, снижения стоимости создания и испытаний ДУ таких МКА, рядом зарубежных организаций выполнены исследования перспективных компонентов топлива и новых схемных решений [2]. Одним из заявленных направлений работ [1, 3] является создание ДУ на закиси азота $\left(\mathrm{N}_{2} \mathrm{O}\right)$.

Закись азота как компонент ракетного топлива нашла широкое применение в работах компании XCOR Aerospace [4]. Начиная с 2000 года по заказу NASA и DARPA, компанией XCOR Aerospace совместно с ATK GASL выполнена разработка и исследование более 10 вариантов ЖРД с диапазоном тяги $10 . .2000$ Н и использованием $\mathrm{N}_{2} \mathrm{O}$ в качестве окислителя. 
Несмотря на возросший интерес к закиси азота в аэрокосмической отрасли, существует ряд вопросов, связанных с эффективностью ее применения для МКА с массой менее 100 кг. Выполненный в работах [1,3] анализ не позволяет однозначно сделать вывод о целесообразности разработки и внедрения ДУ на закиси азота в структуру перспективных МКА. В литературных источниках не показана количественная оценка снижения массогабаритных параметров за счет применения $\mathrm{N}_{2} \mathrm{O}$, а также не выполнено сравнение с существующими образцами ДУ МКА.

В настоящей работе сделана попытка определения границ области применения и количественного показателя эффективности ДУ на закиси азота для МКА класса "Микро".

Статистические данные, приведённые на рис.1, демонстрируют рост доли сухой массы

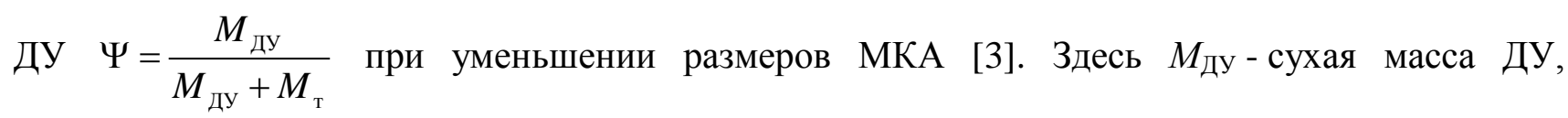

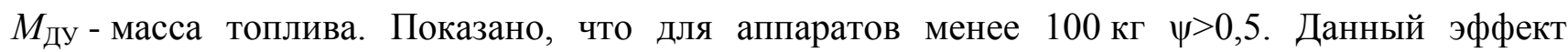
приводит к существенному снижению массовой энергоотдачи ДУ $\eta_{\mathrm{m}}=\frac{I_{\text {п }}}{M_{\text {ду }}+M_{\text {т }}}$. Здесь

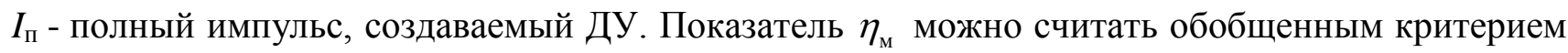
массовой эффективности ДУ МКА.

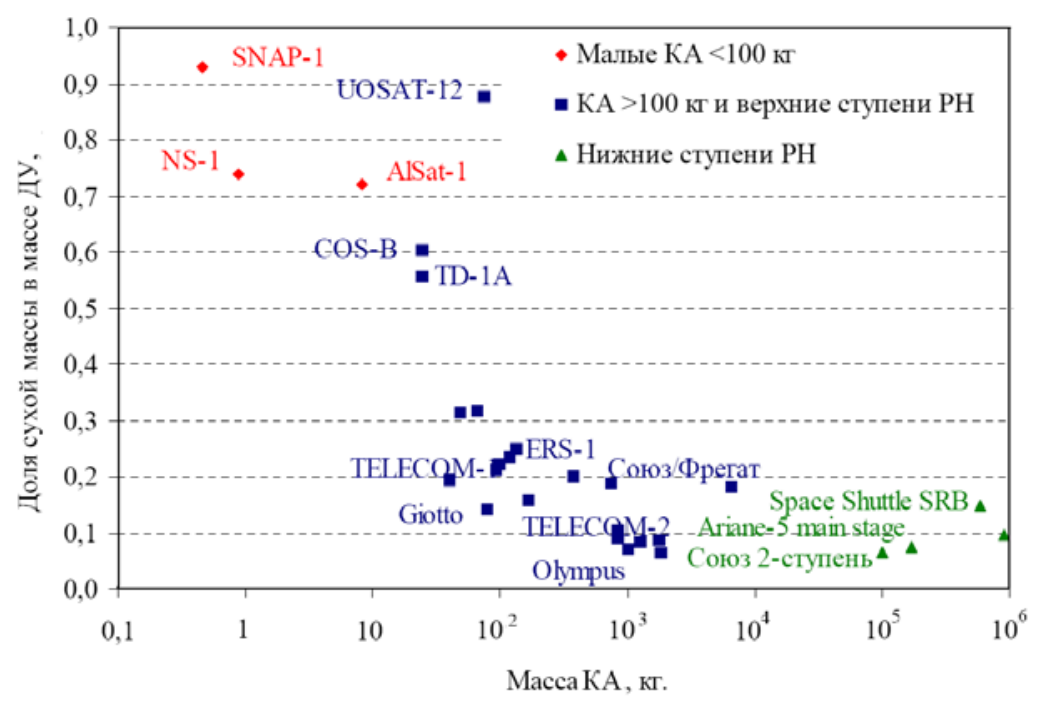

Рис. 1. Доля сухой массы КА различного назначения

Одной из главных причин роста сухой массы ДУ с уменьшением линейных размеров МКА является то, что толщины конструктивных элементов и размеры агрегатов при достижении минимального, технологически возможного, предельного значения остаются постоянными и не подлежат дальнейшему масштабированию.

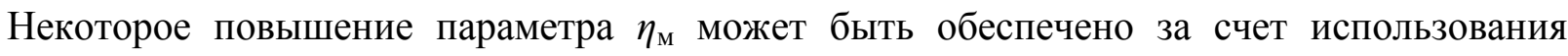
высокоэнегетических одно- или двухкомпонентных топлив с высокими плотностями. Однако в большинстве случаев, их применение требует наличия массозатратных систем хранения и вытеснения топлива из баков, внедрения устройств инициации рабочего процесса. Кроме того, 
создание ракетного двигателя (РД) с реализацией эффективного рабочего процесса при номинальном уровне тяги менее $0,5 \mathrm{H}$ требует решения многих технических проблем, что приводит к увеличению массы конструкции.

Согласно информации, полученной на основе анализа открытых данных, начиная с 1970 г до настоящего времени [2, 5] можно выявить вполне определенную тенденцию (рис. 2), характеризующую изменение уровня массовой энергоотдачи ДУ МКА различного назначения. При анализе рассмотривались МКА с массой до 500 кг и характеристической скоростью $u_{\text {хар }}$ до $100 \mathrm{~m} / \mathrm{c}$.

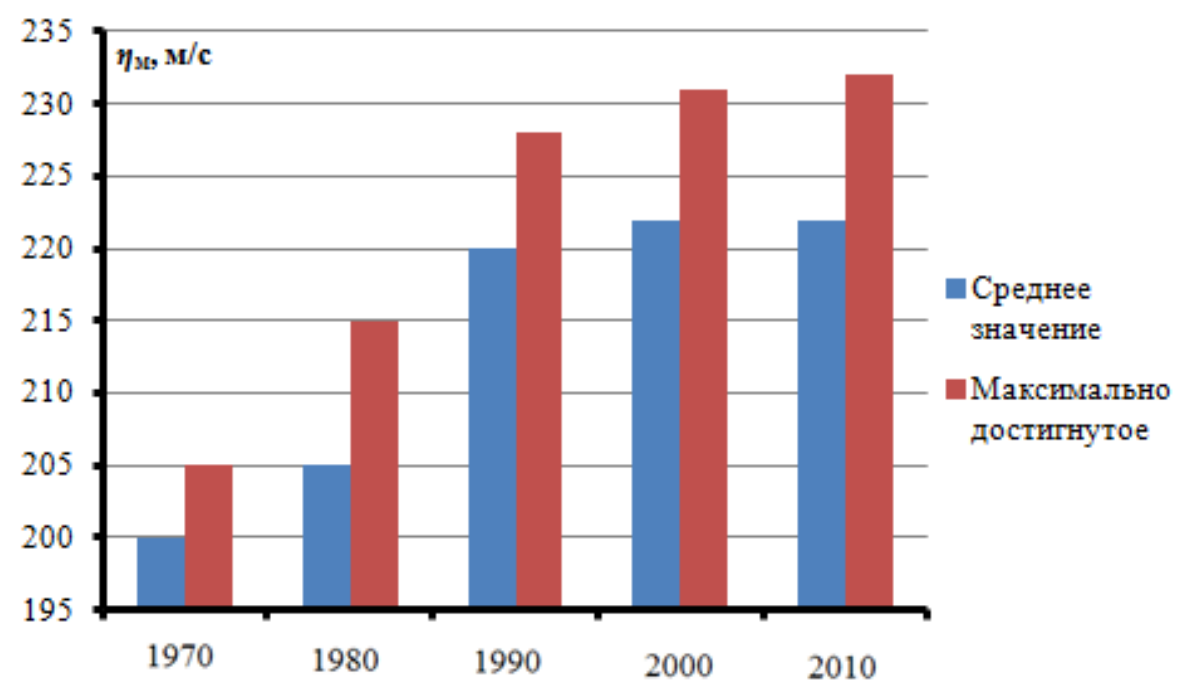

Рис. 2. Показатели массовой энергоотдачи для МКА за период 1970-2010 гг

Основываясь на работах [2, 5], для МКА массой от 1 до 100 кг с комбинированными ДУ, включающими ЖРД или РДТТ, можно построить осредненный график (рис. 3), характеризующий достигнутый мировой уровень показателя $\eta_{\mathrm{M}}$ при различных характеристических скоростях МКА.

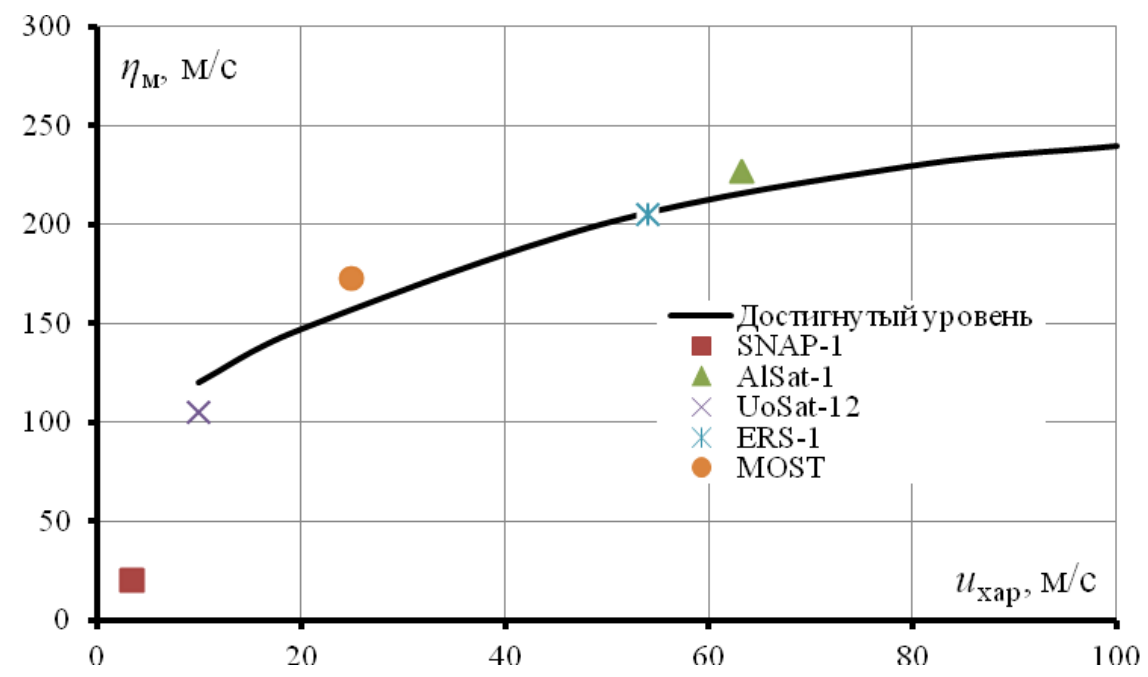

Рис. 3. Массовая энергоотдача ДУ МКА массой от 10 до 20 кг 
Рис. 2 демонстрирует существенное снижение темпов роста массовой энергоотдачи ДУ

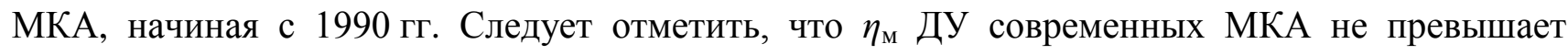
$250 \mathrm{M} / \mathrm{c}$ (рис. 3).

Для дальнейшего повышения показателя $\eta_{\text {м }}$ требуется не только внедрение перспективных компонентов топлива, но и реализация схемных решений, позволяющих существенно снизить сухую массу ДУ МКА.

Как упоминалось выше, одним из направлений, позволяющих повысить массовую энергоотдачу ДУ МКА является применение закиси азота. Наиболее важными преимуществами $\mathrm{N}_{2} \mathrm{O}$ являются возможность разложения ее на свободные кислород и азот с выделением тепловой энергии в количестве 82 кДж/моль, возможность хранения в сжиженном состоянии, упрощение системы подачи за счет эффекта самовытеснения собственными насыщенными парами с давлением более 4 МПа при 290 К, а также нетоксичность.

Совокупность вышеизложенных свойств закиси азота позволяет разработать ДУ МКА на этом компоненте топлива, сочетающую в себе технологичность конструкции и эксплуатации, надежность и многорежимность. Некоторые из возможных вариантов схем ДУ МКА представлены на рис. 4.

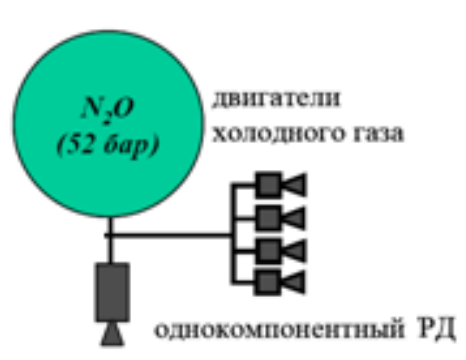

A)

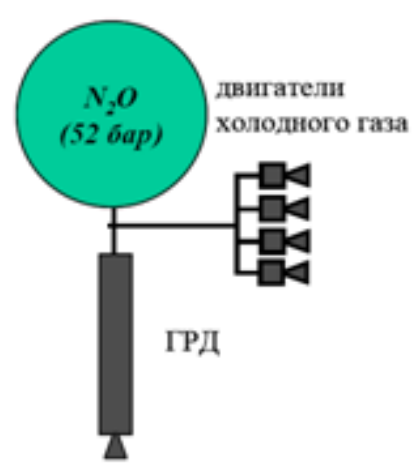

Б)

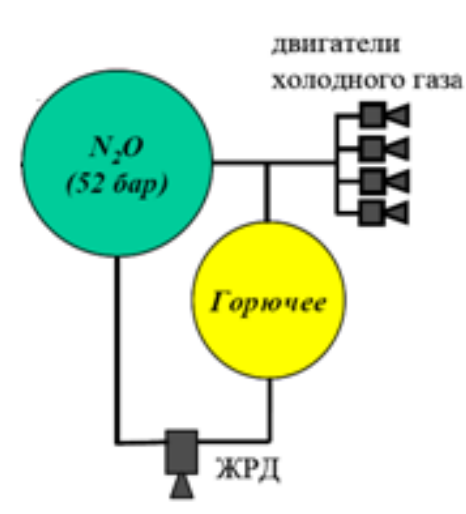

B)

Рис. 4. Схемы ДУ на закиси азота с использованием в качестве маршевого двигателя однокомпонентного РД (а), ГРД (б) и ЖРД (в)

Подобные ДУ состоят из маршевых РД с уровнем тяги $P_{\mathrm{M}}=5 . .500$ Н и управляющих РД с уровнем тяги $P_{\mathrm{y}}<1 \mathrm{H}$. В рассматриваемых вариантах управляющими являются РД на холодном газе, в то время как в качестве маршевых могут выступать однокомпонентный РД с каталитическим разложением закиси азота, гибридный РД (ГРД) или жидкостный РД (ЖРД). Работы [3, 6, 7] показывают возможность создания однокомпонентных и двухкомпонентных ЖРД удовлетворяющих требованиям, предъявляемым к элементам ДУ МКА по уровню тяги, частоте и скважности включений, ресурсу, импульсу последействия а также экологической безопасности. 
Целью работы является определение области эффективного применения систем на закиси азота в ДУ МКА (применительно к классу МКА "микро").

С этой целью проведено сравнение по значению $\eta_{\mathrm{m}}$ приведенных выше вариантов ДУ на закиси азота (рис. 4) с аналогами на гидразине (рис. 5a), газообразных $\mathrm{O}_{2} / \mathrm{H}_{2}$ (рис. 5б), НДМГ/АТ (рис. 5в) и РД на холодном газе (рис. 5г). Анализ выполнен при заданных значениях тяги маршевых РД $\left(P_{\mathrm{M}}=50 \mathrm{H}\right)$ и управляющих РД $\left(P_{\mathrm{y}}=0,5 \mathrm{H}\right)$. В качестве исследуемой параметрической области взят диапазон массы МКА $\mathrm{M}_{\text {КА }}=10 . . .100$ кг с полным импульсом ДУ $I_{\Pi}=10 \ldots 5000 \mathrm{H}^{*} \mathrm{c}$. Принято, что $60 \%$ полного импульса приходится на маршевые РД и 40 \% на управляющие.

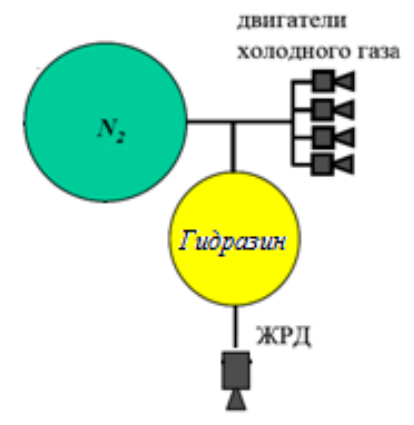

a

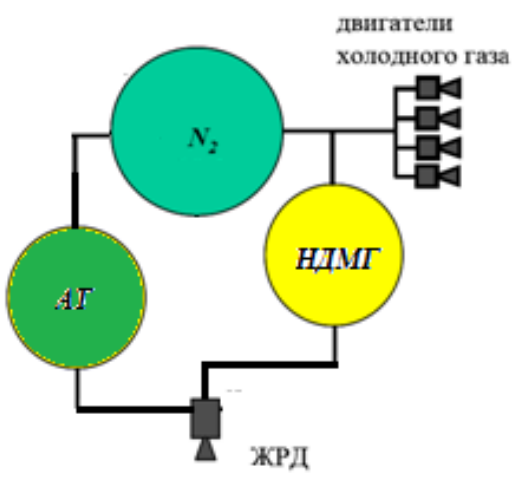

B

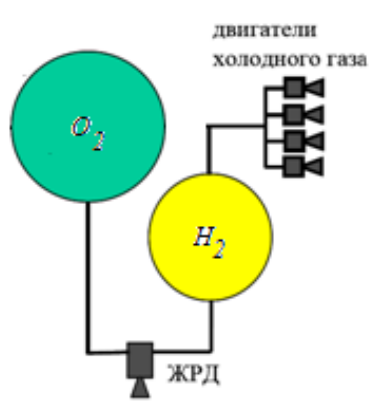

6

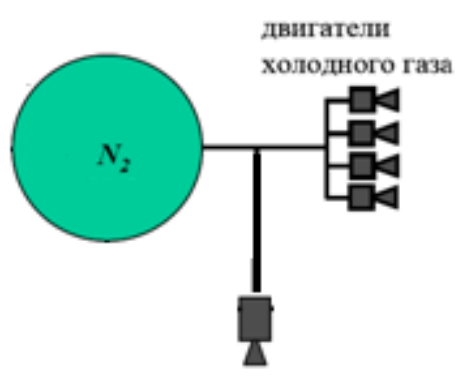

$\Gamma$

Рис. 5. Схемы ДУ на гидразине (а), газообразных кислороде и водороде (б), АТ/НДМГ (в) и на "холодном газе" (в)

Расчетное определение $\eta_{\mathrm{M}}$ проводилось в соответствии с [8]. Масса топлива определялась как $M_{\mathrm{r}}=\frac{0,6 I_{\text {п }}}{I_{\mathrm{y}}^{\mathrm{M}}}+\frac{0,4 I_{\text {п }}}{I_{\mathrm{y}}^{\mathrm{y}}}$, где $I_{\mathrm{y}}^{\mathrm{M}}-$ удельный импульс маршевого РД и $I_{\mathrm{y}}^{\mathrm{y}}-$ удельный импульс управляющего РД. Масса ДУ вычислялась как сумма масс элементов $M_{\text {ду }}=\sum_{i=1}^{N} M_{\mathrm{i}}$. Агрегаты вариантов ДУ выбирались с учетом существующих и перспективных схемных решений [8]. 
Масса $M_{\mathrm{i}}$ каждого агрегата ДУ соответствует номенклатуре, производимых в РФ элементов ДУ [9-11]. Удельный импульс РД в пустоте $\left(I_{\text {уп }}\right)$ выбран в соответствии с таблицей 2.

Таблица 2. Удельный импульс РД

\begin{tabular}{|c|c|c|c|}
\hline Тип РД, топливо & $I_{\text {уп }}$ м/с & Тип РД, топливо & $I_{\text {уп }}, \mathrm{m} / \mathrm{c}$ \\
\hline Однокомпонентный РД на $\mathrm{N}_{2} \mathrm{O}$ & 1950 & ЖРД на $\mathrm{H}_{2}$ и $\mathrm{O}_{2}$ & 2960 \\
\hline $\begin{array}{c}\text { ГРД на бутадиеновом горючем } \\
\text { и } \mathrm{N}_{2} \mathrm{O}\end{array}$ & 2070 & ЖРД на НДМГ и АТ & 2510 \\
\hline $\begin{array}{c}\text { ЖРД на этаноле и } \mathrm{N}_{2} \mathrm{O} \\
\text { Однокомпонентный РД на } \\
\text { гидразине }\end{array}$ & 2210 & РД на "холодном газе" $\left(\mathrm{N}_{2} \mathrm{O}\right)$ & 510 \\
\hline
\end{tabular}

В результате проведенных расчетов определены значения массовой энергоотдачи в зависимости от полного импульса ДУ МКА (рис. 6).

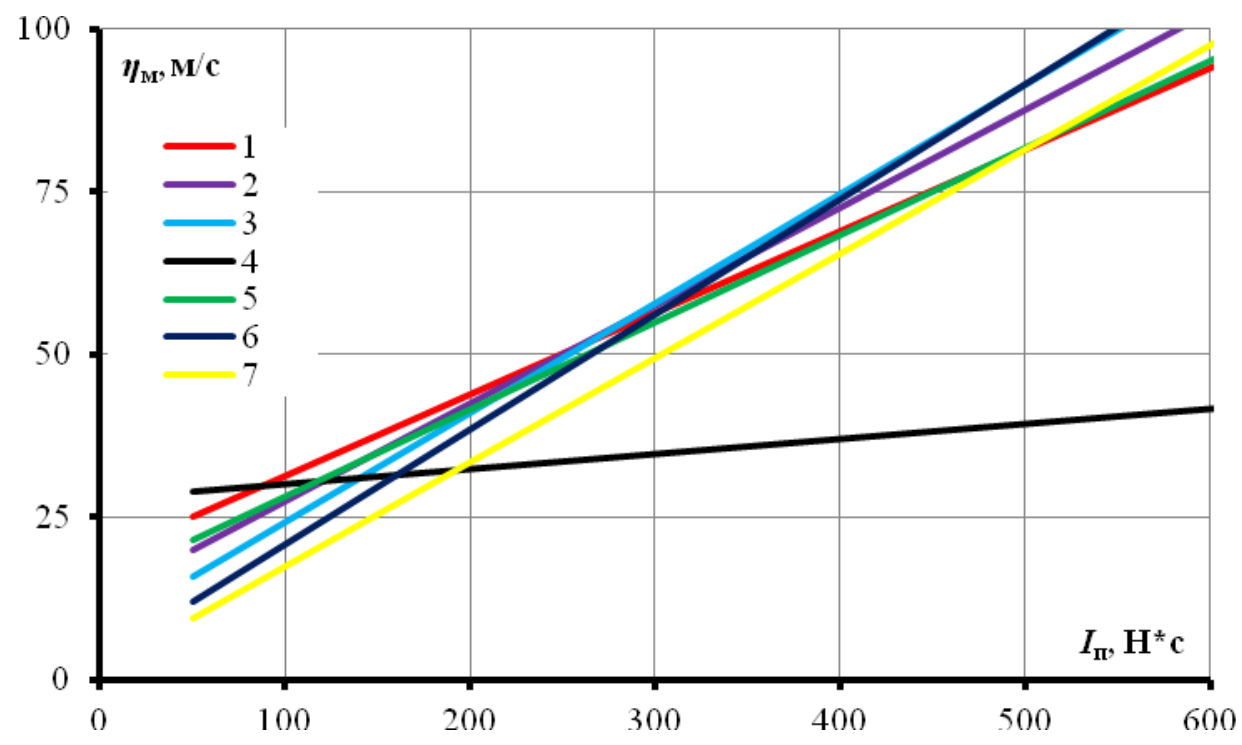

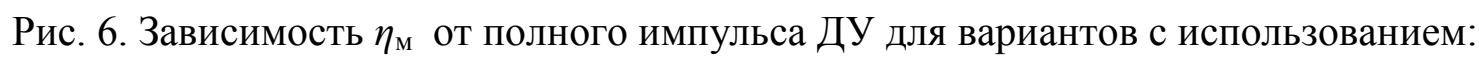
однокомпонентного РД на $\mathrm{N}_{2} \mathrm{O}$ (1), ГРД на бутадиеновом горючем и $\mathrm{N}_{2} \mathrm{O}(2)$, ЖРД на этаноле и $\mathrm{N}_{2} \mathrm{O}$ (3), ДУ на "холодном газе" (4), однокомпонентном РД на гидразине (5), РД на газообразных $\mathrm{H}_{2}$ и $\mathrm{O}_{2}(6), \mathrm{AT} / \mathrm{HДМГ} \mathrm{(7)}$

На основе выполненного анализа можно сделать следующие выводы. Применение ДУ на закиси азота позволяет снизить массу МКА, если диапазон значений полного импульса ДУ составляет $120 \ldots 470 \mathrm{H}^{*}$ с. При $I_{\Pi}<260$ м/с более целесообразным становится применение ДУ с 
однокомпонентным маршевым РД на $\mathrm{N}_{2} \mathrm{O}$, а при $I_{\Pi}>260 \mathrm{M} / \mathrm{c}$ - двухкомпонентного ЖРД с использованием $\mathrm{N}_{2} \mathrm{O}$ в качестве окислителя. Расчетными методами показано, что применение закиси азота дает возможность снижения массы ДУ МКА на 10...15\% относительно ДУ традиционных схем, применяемых в настоящее время.

\section{Список литературы}

1. Zakirov V.A., Wan K., Tang C., Shan F., Zhang H., Li L. $\mathrm{N}_{2} \mathrm{O}$ Propulsion Research at Tsinghua: 2006 // Proceedings of ESA Space Propulsion Conference, 3nd International Conference on Green Propellants for Space Propulsion, SP-635. Poitiers. France, 17-20 September 2006. P. 128-132.

2. Банго В.И., Разумовский Ю.К., Зайцев И.В. Анализ современных тенденций и направлений развития малых космических аппаратов за рубежом // 3-я Международная конференция-выставка «Малые спутники, новые технологии, миниатюризация. Области эффективного применения в XXI веке». г. Королев, Московская обл., 27-31 мая 2002. Кн.1. С. 381-387.

3. Zakirov V.A., Lawrence T.J., Sellers J.J., Sweeting M.N. Nitrous Oxide as a Rocket Propellant // Proceedings of the 51st International Astronautical Congress. Rio de Janeiro, Brazil, 2-6 October 2000. (Also published in: Acta Astronautica. 2001. Vol. 48, no. 5-12. P. 353-362. DOI: http://dx.doi.org/10.1016/S0094-5765(01)00047-9 ).

4. Xcor Aerospace: [Электронный ресурс]. Режим доступа: http://www.xcor.com (дата обращения 10.05.2012).

5. Лукьященко В.И., Саульский В.К., Шучев В.А, Смирнов В.В. Международные тенденции создания и эксплуатации малых космических аппаратов // 3-я Международная конф.-выставка «Малые спутники, новые технологии, миниатюризация. Области эффективного применения в XXI веке». г. Королев, Московская обл., 27-31 мая 2002. Кн.1. С. 332-348.

6. Tiliakos N., Tyll J.S., Herdy R., Sharp D., Moser M., Smith N. Development and Testing of a Nitrous Oxide/Propane Rocket Engine, AIAA2001-3834 // 37th AIAA/ASME/SAE/ASEE Joint Propulsion Conference. Salt Lake City, Utah, 8-11 July 2001.P. 258-264.

7. Zakirov V.A., Ke Wan, Fan-li Shan, Hai-yun Zhang, Lu-ming Li. Restartable Hybrid Rocket Motor using Nitrous Oxide, Paper IAC-06-C4.2.02 // Proceedings of 57th International Astronautical Congress. Valencia, Spain, 2-6 October 2006, P. 92-101.

8. Добровольский М.В. Жидкостные ракетные двигатели. М.: МГТУ им. Н.Э Баумана, 2005. 486 с.

9. Официальный сайт ФГУП ОКБ «Факел»: [Электронный ресурс]. Режим доступа: http://www.fakel-russia.com/ (дата обращения 10.05.2012).

10. Официальный сайт КБхиммаш им. А.М. Исаева: [Электронный ресурс]. Режим доступа: http://www.kbhmisaeva.ru (дата обращения 10.05.2012). 
11. Официальный сайт ФГУП НИИМАШ: [Электронный ресурс]. Режим доступа: http://niimashspace.ru (дата обращения 10.05.2012). 


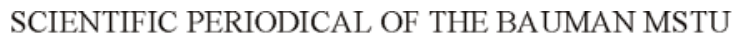 \\ SCIENCE and EDUCATION
}

EL No FS77 - 48211. No0421200025. ISSN 1994-0408

electronic scientific and tec hn i c a j j o r n a l

\section{Analysis of effective use of nitrous oxide as a fuel component for propulsion systems of small spacecrafts \\ \# 09, September 2012 \\ DOI: 10.7463/0912.0450400 \\ Voroneckii A.V., Aref'ev K.Yu.}

\begin{abstract}
Russia, Bauman Moscow State Technical University
voron@mx.bmstu.ru

arefyev@rambler.ru
\end{abstract}

The paper presents the results of a system analysis of efficiency of nitrous oxide $\left(\mathrm{N}_{2} \mathrm{O}\right)$ as a fuel component for small spacecrafts (SSC). The authors define the criterion for mass efficiency of the propulsion system (PS) of small spacecrafts. They show the current global level of development of SSC remote control. Application field of nitrous oxide in SSC remote control was calculated, and mass efficiency of $\mathrm{N}_{2} \mathrm{O}$ application was quantitatively defined.

Publications with keywords:system analysis, microsatellite, propulsion, nitrous oxide Publications with words:system analysis, microsatellite, propulsion, nitrous oxide

\section{References}

1. Zakirov V.A., Wan K., Tang C., Shan F., Zhang H., Li L. N2O Propulsion Research at Tsinghua: 2006. Proceedings of ESA Space Propulsion Conference, 3nd International Conference on Green Propellants for Space Propulsion, SP-635. Poitiers. France, 17-20 September 2006, pp. 128-132.

2. Bango V.I., Razumovskii Iu.K., Zaitsev I.V. Analiz sovremennykh tendentsii i napravlenii razvitiia malykh kosmicheskikh apparatov za rubezhom [Analysis of current trends and directions of development of small spacecraft abroad]. 3-ia Mezhdunarodnaia konferentsiiavystavka «Malye sputniki, novye tekhnologii, miniatiurizatsiia. Oblasti effektivnogo primeneniia $v$ 21 veke» [The 3rd international conference-exhibition «Small satellites, new technologies, miniaturization. The fields of effective use in the 21 century»]. Korolev, Moscow Region, 27-31 May 2002, vol.1, pp. 381-387.

3. Zakirov V.A., Lawrence T.J., Sellers J.J., Sweeting M.N. Nitrous Oxide as a Rocket Propellant. Proceedings of the 51st International Astronautical Congress. Rio de Janeiro, Brazil, 2-6 October 2000. (Also published in: Acta Astronautica. 2001, vol. 48, no. 5-12, pp. 353362. DOI: http://dx.doi.org/10.1016/S0094-5765(01)00047-9). 
4. Xcor Aerospace. Available at: http://www.xcor.com , accessed 10.05.2012.

5. Luk'iashchenko V.I., Saul'skii V.K., Shuchev V.A, Smirnov V.V. Mezhdunarodnye tendentsii sozdaniia i ekspluatatsii malykh kosmicheskikh apparatov. 3-ia Mezhdunarodnaia konferentsiia-vystavka «Malye sputniki, novye tekhnologii, miniatiurizatsiia. Oblasti effektivnogo primeneniia $v 21$ veke» [The 3rd international conference-exhibition «Small satellites, new technologies, miniaturization. The fields of effective use in the 21 century»]. Korolev, Moscow Region, 27-31 May 2002, vol.1, pp. 332-348.

6. Tiliakos N., Tyll J.S., Herdy R., Sharp D., Moser M., Smith N. Development and Testing of a Nitrous Oxide/Propane Rocket Engine. AIAA2001-3834. 37th AIAA/ASME/SAE/ASEE Joint Propulsion Conference. Salt Lake City, Utah, 8-11 July 2001, pp. 258-264.

7. Zakirov V.A., Ke Wan, Fan-li Shan, Hai-yun Zhang, Lu-ming Li. Restartable Hybrid Rocket Motor using Nitrous Oxide. Paper IAC-06-C4.2.02. Proceedings of 57th International Astronautical Congress. Valencia, Spain, 2-6 October 2006, pp. 92-101.

8. Dobrovol'skii M.V. Zhidkostnye raketnye dvigateli [Liquid rocket engines]. Moscow, Bauman MSTU Publ., 2005. 486 p.

9. Ofitsial'nyi sait FGUP OKB “Fakel” [Official website of the Federal State Unitary Enterprise "Experimental Design Bureau "Fakel"]. Available at: http://www.fakel-russia.com , accessed 10.05.2012.

10. Ofitsial'nyi sait KBkhimmash im. A.M. Isaeva [Official website of the Design Bureau of Chemical Engineering of them. A.M. Isaev]. Available at: http://www.kbhmisaeva.ru , accessed 10.05.2012.

11. Ofitsial'nyi sait FGUP NIIMASh. [Official website of the Federal State Unitary Enterprise "Research Institute of Mechanical Engineering”]. Available at: http://niimashspace.ru , accessed 10.05.2012. 